\title{
Levantamento florístico das Rodofíceas do Arquipélago de São Pedro e São Paulo (ASPSP) - Brasil ${ }^{1}$
}

\author{
Douglas Correia Burgos², Sonia Maria Barreto Pereira ${ }^{2,3}$ e Maria Elizabeth Bandeira-Pedrosa ${ }^{3}$
}

Recebido em 15/05/2008. Aceito em 31/03/2009

RESUMO - (Levantamento florístico das rodofíceas do Arquipélago de São Pedro e São Paulo (ASPSP) - Brasil). Este trabalho apresenta o levantamento

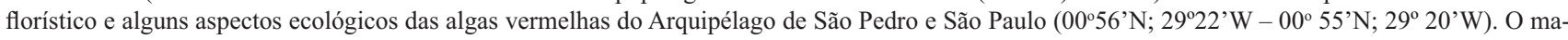
terial foi coletado durante as marés baixas em seis estações, nas zonas entre-marés e infralitoral no mês de outubro de 2003 (estação seca) e abril de 2005 (estação chuvosa). Foram registradas 24 espécies, distribuídas entre as ordens Bonnemaisoniales (uma espécie), Ceramiales (12 espécies), Corallinales (seis espécies), Erythropeltidales (uma espécie), Gelidiales (uma espécie), Rhodymeniales (duas espécies) e Stylonematales (uma espécie). Foram feitos 15 novos registros de rodofíceas à flora do Arquipélago e quatro novas adições para as ilhas oceânicas brasileiras: Ceramium brevizonatum var. caraibicum H. E. Petersen \& Börgesen, Ceramium tenerrimum (G. Martens) Okamura, Jania prolifera A. B. Joly e Stylonema alsidii (Zanardini) K. M. Drew. Foram registradas algas vermelhas em apenas três estações. A maior riqueza de táxons (16 taxa) ocorreu na Estação 01 (Enseada) e no período chuvoso (23 taxa). A similaridade florística, com base no índice de Sørensen, em relação à profundidade, variou de 0 a $0.66 \%$.

Palavras-chave: Algas marinhas, Rhodophyta, Arquipélago, Brasil

ABSTRACT - (Floristic survey of rhodophycean of the São Pedro and São Paulo Archipelago (ASPSP) - Brazil). This paper presents a floristic survey of the red algae and some ecological aspects of the São Pedro and São Paulo Archipelago ( $\left.00^{\circ} 56^{\prime} \mathrm{N} ; 29^{\circ} 22^{\prime} \mathrm{W}-00^{\circ} 55^{\prime} \mathrm{N} ; 2^{\circ} 20^{\prime} \mathrm{W}\right)$. Collections were carried out during low tide in six stations located in the intertidal and infralittoral zones, in October 2003 (dry season) and April 2005 (rainy season). It was registered 24 infrageneric taxa distributed among the orders Bonnemaisoniales ( 1 specie), Ceramiales (12 species), Corallinales (6 species), Erythropeltidales (1 specie), Gelidiales (1 specie), Rhodymeniales ( 2 species) and Stylonematales (1 specie). Fifteen taxa are new records to the Archipelago flora. It was also registered four new records to the Brazilian oceanic islands: Ceramium brevizonatum var. caraibicum $\mathrm{H}$. E. Petersen \& Börgesen, Ceramium tenerrimum (G. Martens) Okamura, Jania prolifera A. B. Joly and Stylonema alsidii (Zanardini) K. M. Drew. The red algae were registered only in three stations. The highest specific diversity (16) occurred to Station 01 (Enseada) and to the rainy season (23 species). The Søresen floristic similarity in relation to depth varied from 0 to $0.66 \%$.

Key words: Seaweed, Rhodophyta, Archipelago, Brazil

\section{Introdução}

Apesar do bom conhecimento sobre a flora ficológica brasileira, permitindo que o Brasil seja considerado líder neste aspecto entre os países da América do Sul, observa-se que o mesmo está concentrado na região entre-marés. Neste aspecto, alguns trabalhos mais recentes merecem destaque como: Oliveira Filho (1969), Yoneshigue-Braga et al. (1986), Bandeira-Pedrosa et al. (2003; 2004), Pereira et al. (2002), Pereira \& Accioly (1998), Muñoz \& Pereira (1997), Mansilla \& Pereira (1998) e Oliveira-Carvalho et al. (2003), Guimarães (2006), Torres et al. (2004) e Pereira et al. (2008)

Devido a onerosidade e dificuldade de acesso a região infralitoral, pouquíssimas pesquisas foram desenvolvidas citando-se entre estas Joly \& Yoneshigue-Braga (1966), Joly \& Oliveira Filho (1968), Joly \& Sazima (1971), Joly et al. (1976), Oliveira Filho (1976), Ugadim \& Pereira (1978), Pereira et al. (1981), Guimarães et al. (1981) e YoneshigueValentin et al. 2005. Em relação às ilhas oceânicas, os estudos são igualmente, reduzidos, citando-se, para uma melhor avaliação, o trabalho de Villaça et al. (2006).

O Brasil possui quatro arquipélagos oceânicos (Fernando de Noronha, Trindade, Martins Vaz e São Pedro e São Paulo) e um atol (Rocas). Dentre estes, o Arquipélago de São Pedro e São Paulo tem grande interesse biogeográfico devido ao isolamento de suas ilhas, sua posição intermediária entre a África Ocidental e a América do Sul e seu reduzido tamanho (Edwards \& Lubbock 1983; Edwards 1984). Por estes motivos, o conhecimento da sua fauna e flora marinha são de grande relevância (Edwards 1984). Do ponto de vista ecológico, apresenta marcada biodiversidade, incluindo a ocorrência de várias espécies endêmicas de peixes, como citado por Lubbock \& Edwards (1981), Feitosa et al. (2003) e Vaske-Jr. et al. (2005).

Sob o ponto de vista ficológico, o Arquipélago de Fernando de Noronha é o mais bem estudado, ao contrário do Arquipélago de São Pedro e São Paulo, onde foi realizado um reduzido número de pesquisas sobre a flora de algas marinhas bentônicas (Dickie 1875; Edwards \& Lubbock 1983; Pereira et al. 2006) e mesmo assim, faltando muito para o real conhecimento de sua flora. Este fato é um motivo de preocupação devido ao isolamento dessas formações e a fragilidade desses ecossistemas. Em geral, os estudos ficológicos das ilhas oceânicas estão baseados, principalmente, nas comunidades que ocorrem na zona entre-marés, o que é bastante limitado para se definir um padrão florístico (Villaça et al. 2006).

A Comissão Interministerial para os Recursos do Mar (CIRM), aprovou em 1996 o Programa Arquipélago de São Pedro e São Paulo (Pró-Arquipélago), criando o grupo de trabalho permanente (GT Arquipélago) visando sua ocupação física e, assim, garantindo a soberania nacional de

\footnotetext{
Parte da Dissertação de Mestrado do primeiro Autor

2 Programa de Pós-Graduação em Botânica (PPGB) - Universidade Federal Rural de Pernambuco

3 Departamento de Biologia Universidade Federal Rural de Pernambuco, Recife, PE, Brasil

Autor para correspondência: burgosdc@yahoo.com.br
} 
200 milhas náuticas ao redor do mesmo, que junto com o Arquipélago de Trindade aumentou em $900 \mathrm{mil} \mathrm{km}^{2}$ a Zona Econômica Exclusiva (ZEE) brasileira. Posteriormente, passou a receber auxílio financeiro do Conselho Nacional do Desenvolvimento Científico e Tecnológico (CNPq). Mesmo assim, continua sendo difícil a realização de pesquisas tendo em vista principalmente, a distância do continente para o arquipélago e ausência de periodicidade para a realização das expedições científicas.

Tendo em vista o reduzido número de trabalhos sobre a flora ficológica do referido arquipélago, este trabalho teve como objetivo iniciar esse inventário pelo filo Rhodophyta, incluindo aspectos ecológicos (batimetria, tipo de substrato e epifitismo) e de distribuição de seus representantes entre as ilhas oceânicas brasileiras e o continente, representado neste trabalho, pelo estado de Pernambuco.

\section{Material e métodos}

Área estudada - o Arquipélago de São Pedro e São Paulo, formado por sete ilhas rochosas, está situado entre as coordenadas $00^{\circ} 56^{\prime} \mathrm{NE} 29^{\circ} 22^{\prime} \mathrm{W}$; $00^{\circ} 55^{\prime} \mathrm{NE} 29^{\circ} 20^{\prime} \mathrm{W}$; ao Norte do Equador sobre a Cadeia Dorsal MesoAtlântica (Vaske-Jr et al. 2006), distando cerca de $1.100 \mathrm{~km}$ da cidade de Natal (RN) e 522 km do Arquipélago de Fernando de Noronha (PE) (Fig. 1).

A extensão da área emersa fica em torno de $17.000 \mathrm{~m}^{2}$ e a distância entre os pontos extremos, que se situa entre as rochas Sacadura Cabral e Graça Aranha, é de $420 \mathrm{~m}$. As ilhotas apresentam contornos sinuosos irregulares, e reentrantes, e suas encostas possuem forte declive $\left(>80^{\circ}\right)$. As quatro ilhotas maiores (Belmonte, São Paulo, São Pedro e Barão de Teffé) estão separadas entre si por estreitos canais, formando uma enseada em forma de ferradura, com dimensões médias de $100 \mathrm{~m}$ de comprimento, 50 $\mathrm{m}$ de largura e $6 \mathrm{~m}$ de profundidade. $\mathrm{O}$ fundo desta enseada é constituído por sedimentos provenientes da atividade biológica e do desagregamento das rochas que constituem o arquipélago. O relevo emerso do ASPSP é acidentado, e seus pontos culminantes situam-se na ilhota São Pedro (18 $\mathrm{m}$ de altitude), na ilhota Belmonte ( $16 \mathrm{~m}$ de altitude) e na ilhota São Paulo (14 m de altitude). É o único arquipélago oceânico de origem não vulcânica (Edwards \& Lubbock 1983). Suas rochas são plutônicas representando o manto superior da crosta oceânica e aflorando na superfície, através de forças tectônicas nas falhas transversais da Cordilheira Meso-Atlântica (Campos et al. 2005).

$\mathrm{O}$ arquipélago recebe influência da corrente marítima Sul Equatorial no sentido Leste-Oeste e da contra-corrente Sul Equatorial em sentido contrário, a qual situa-se de $60 \mathrm{~m}$ a $100 \mathrm{~m}$ de profundidade abaixo da primeira

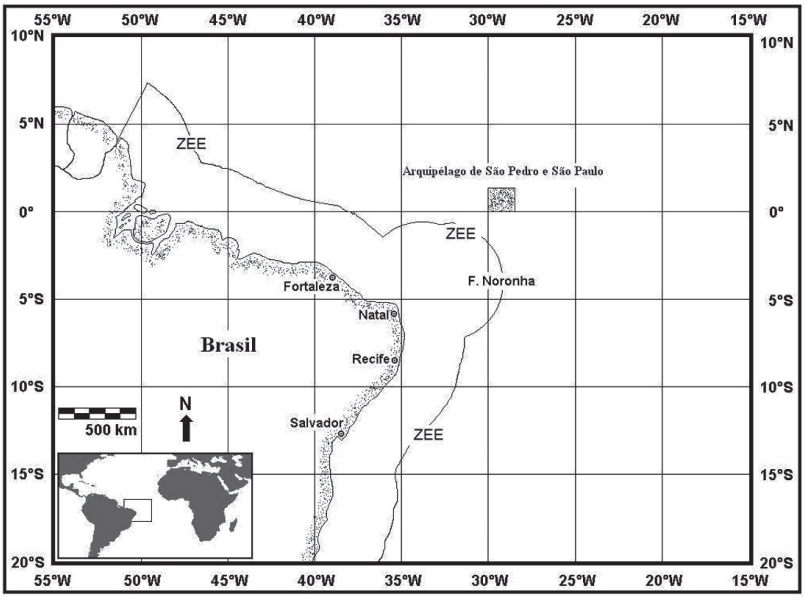

Figura 1. Mapa com a localização do Arquipélago de São Pedro e São Paulo, Brasil (Travassos et al. 1999, modificado).
(Travassos et al. 1999). Ocorrem dois períodos sazonais, um chuvoso de março a julho com temperatura do ar em torno de $24^{\circ} \mathrm{C}$ e o outro seco de setembro a fevereiro com temperatura em torno de $28^{\circ} \mathrm{C}$ (dados cedidos pelo Instituto Nacional de Meteorologia).

Parte do habitat terrestre da área encontra-se a quatro metros acima do nível do mar, recebendo constantemente influxo da água proveniente das ondas ou de respingos. Existem nesta área diversas poças ou piscinas naturais onde são encontrados diferentes organismos como: cnidários, poríferos, moluscos, equinodermos, crustáceos, briozoários, peixes e algas. O sub-litoral do arquipélago, apresenta um declive acentuado atingindo até $60 \mathrm{~m}$ de profundidade, distinguindo-se: Zona Palythoa, distribuída de 0-5 $\mathrm{m}$ de profundidade; Zona Caulerpa racemosa, dominando a partir de 5 $\mathrm{m}$ até os $33 \mathrm{~m}$; Zona Sub-caulerpa, subjacente a anterior e é dominada por invertebrados marinhos e Áreas de substratos não consolidados, que se constitui de poucas porções de sedimento grosseiro e de pedregulhos (Edwards \& Lubbock 1983).

Coleta e análise dos dados - com base em mapas e fotos do arquipélago, foram plotadas, seis estações de coletas em locais de fácil acesso. A Estação 01 está localizada na enseada do arquipélago, parcialmente, abrigada em forma de ferradura, apresenta fundo com substrato consolidado, com batimetria mínima de 4 metros, logo abaixo do píer e máxima de 23 metros, na boca da enseada. A Estação 02 situa-se na Ilha Challenger, por trás da Estação Científica na região entre-marés, onde ocorre uma zona de forte arrebentação. A Estação 03 encontra-se localizada a oeste da Ilha Challenger, no paredão com declive $\geq 85 \%$ com batimetria acima dos $70 \mathrm{~m}$. A Estação 04 situa-se na Ilha Cabral, no paredão com declive $\geq 85 \%$ com batimetria acima dos $70 \mathrm{~m}$. A Estação 05 localiza-se na área externa do Arquipélago, no local denominado de "Cabeço do Tartaruga", formado por um pico rochoso submerso, exposto a forte movimentação das correntes, sujeita a constante herbivoria (Pereira et al. 2006), com batimetria mínima de 12 metros e máxima de 45 metros na face norte e 55 metros na face sul . A Estação 06, denominada "Rocha Gago Coutinho", encontra-se ao sul da Ilha Challenger, numa área de intensa turbulência, onde apenas sua porção distal encontra-se emersa com profundidade máxima de 15 metros (Fig. 2).

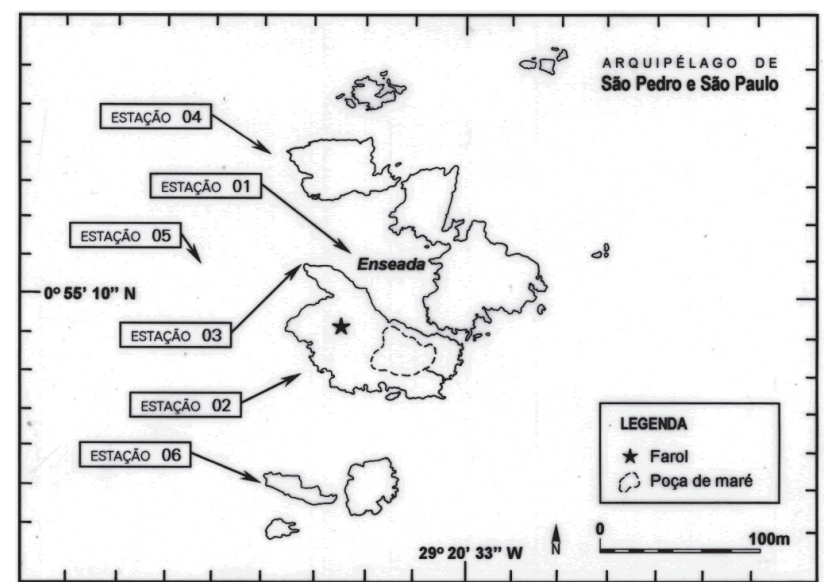

Figura 2. Mapa com a localização das estações de coletas no Arquipélago de São Pedro e São Paulo, Brasil (Edwards \& Lubbock 1983, modificado).

Foram realizadas duas expedições, uma durante o período seco (outubro de 2002) e a outra durante o período chuvoso (abril de 2005) em marés baixa de sizígia. A duração de cada expedição foi de 28 dias, dos quais 15 foram de coleta de campo. Em cada estação de coleta, as amostragens foram feitas até a profundidade máxima possível por estação, com tempo médio de fundo de 20 minutos. Sendo na Estação 01 realizado amostragens até a isóbata de 23 metros, totalizando 45 amostras. Na Estação 02, única localizada no mesolitoral, foram realizadas coletas numa área de dois metros por dois metros, obtendo-se 15 amostras. Na Estação 03, foram realizadas coletas até a profundidade máxima de 45 metros, totalizando 45 amostras. $\mathrm{Na}$ Estação 04 foram realizadas coletas até 45 metros, totalizando 45 amostras. Na Estação 05 foram realizadas 15 coletas entre as isóbatas de 12 a 25 metros, com 15 amostras. Na Estação 06 foram obtidas 15 amostras até a profundidade de máxima de 15 metros. A cada metro o material foi coletado com auxílio de espátula, através de mergulhos autônomos e/ou 
livres, utilizando-se o método de coletas aleatórias tendo sido a batimetria aferida por profundímetro marca Dacor sherwood scuba.

Em campo, as algas foram fixadas em solução de formol a (4\%) em água do mar, acondicionadas em sacos plásticos devidamente etiquetados. Foram feitas algumas observações ecológicas e biológicas quanto ao tipo de substrato, profundidade, epifitísmo e estágio de reprodução. Após a identificação do material as algas foram taxonomicamente posicionadas de acordo com Wynne (2005). O material foi incorporado ao Herbário Prof. Vasconcelos Sobrinho (PEUFR) da Universidade Federal Rural de Pernambuco.

As rodofíceas foram analisadas ainda quanto a batimetria, período de coleta e a ocorrência entre as estações. Para análise comparativa do número de rodofíceas entre as ilhas oceânicas comparou-se os táxons citados para o Arquipélago de Fernando de Noronha por Dickie (1875), Hemsley (1885), Murray (1891), Williams \& Blomquist (1947), Széchy et al. (1987; 1989), Pedrini et al. (1992), Pereira et al. (1996), Pereira et al. (2002), Pereira (2006); para Trindade e Martin Vaz por Richardson (1975), Joly (1950; 1953), Pedrini et al. (1989), Yoneshigue-Valentin et al. (2005) e Villaça et al. (2006); para São Pedro e São Paulo por Hemsley (1885), Edwards \& Lubbock (1983), Pereira et al. (2005) e Pereira (2006) e, finalmente, para o Atol das Rocas citados por Oliveira Filho \& Ugadim (1974; 1976), Barbosa et al. (2003), Fujii \& Villaça (2003) e Villaça et al. (2006).

A diferença no número de espécies registradas entre cada período de coleta foi verificada pelo teste Qui-quadrado, com correção de Yates (Zar 1996). A similaridade florística entre as estações e profundidades foi avaliada pelo Índice de Sørensen e a existência de grupos florísticos entre as rodofíceas das ilhas oceânicas foi obtida pela análise de agrupamento usando o Índice de Sorensen Bray-Curtis e Média de Grupo através do Programa PC-ORD (McCune \& Mefford 1999). A casualidade da análise de agrupamento foi testada pelo método de permutação Monte Carlo (Manly 1997) com 1000 e 2000 replicações respectivamente, através do programa RandMat versão 1.0 (Sérgio Rosso, capturado em setembro de 2003 -http:// eco.ibi.usp.br/labmar/software.htm).

\section{Resultados e discussão}

As rodofíceas estiveram representadas por 24 espécies, distribuídas entre as ordens Bonnemaisoniales (uma espécie), Ceramiales (12 espécies), Corallinales (seis espécies), Erythropeltidales (uma espécie), Gelidiales (uma espécie), Rhodymeniales (duas espécies) e Stylonematales (uma espécie) (Tab. 1). A ordem com maior percentual de representantes foi Ceramiales (48\%), seguida por Corallinales (28\%), Rhodymeniales ( $8 \%$ ), enquanto as demais representadas por $4 \%$ (Fig. 3). A ordem Ceramiales foi representada pelas famílias Ceramiaceae, Delesseriaceae e Rhodomelaceae, sendo que a primeira delas apresentou o maior número de espécies. A referida ordem como a mais representativa, correspondeu

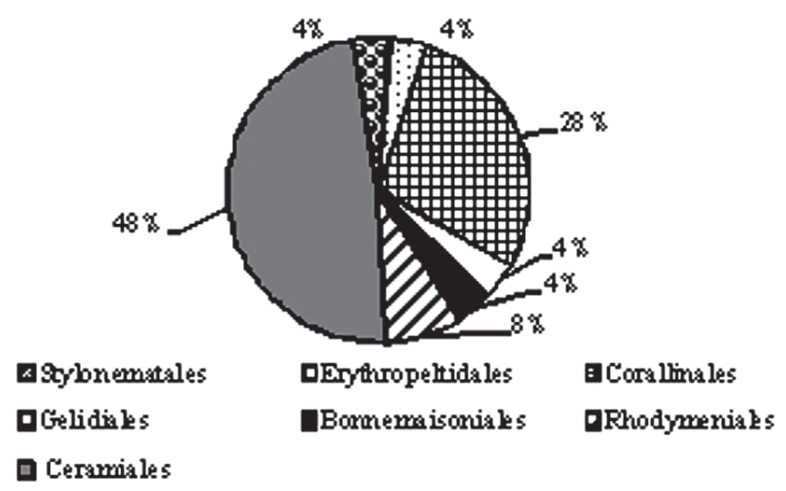

Figura 3. Percentual de distribuição entre as ordens de rodofíceas ocorrentes no Arquipélago de São Pedro e São Paulo, Brasil em outubro de 2002 e abril de 2005. ao que foi encontrado nos trabalhos de Pedrini et al. (1992) e Pereira et al. (2002) para Fernando de Noronha, Pedrini et al. (1989) para a Ilha de Trindade, Oliveira Filho \& Ugadim (1976) para o Atol das Rocas.

Anteriormente, a flora do arquipélago encontrava-se representada por 16 espécies de rodofíceas: Champia parvula (C. Agardh) Harv., Gelidiopsis planicaulis (W.R. Taylor) W.R. Taylor, Hildenbrandia rubra (Sommerf.) Menegh., Hypoglossum hypoglossoides (Stackh.) Collins \& Herv., Nitophyllum wilkinsoniae Collins \& Herv., Jania adhaerens J.V. Lamour., J. capillacea Harv., J. pumila J. V. Lamour. , J. rubens (L.) J.V. Lamour., Laurencia caraibica P. C. Silva, L. filiformis (C. Agardh) Mont., L. microcladia Kütz., Mesophyllum lichenoides (Ellis) Lemoine, Neosiphonia ferulacea Suhr ex J. Agardh, Phymatoliton purpureum (P. Crouan \& H. Crouan) Woelk. \& L. M. Irvine e Polysiphonia. subtilissima Mont. (Hemsley 1885; Edwards \& Lubbock 1983; Pereira et al. 2005). Com este trabalho, foram feitas 15 adições à flora local, das quais, quatro espécies (Ceramium brevizonatum var. caraibicum Ceramium tenerrimum, Jania prolifera A. B. Joly e Stylonema alsidii (Zanardini) K. M. Drew, são novas adições para a flora das ilhas oceânicas brasileiras (Tab. 1). Desta forma, atualmente a flora de rodofíceas no referido arquipélago encontra-se representada por 31 espécies.

Pereira (2006) registrou por engano 19 espécies de rodofíceas para o arquipélago, com base nos trabalhos já citados anteriormente (Hemsley, 1885; Edwards \& Lubbock, 1983 e Pereira et al. 2005). Analisando estes referidos trabalhos foram encontradas 16 espécies.

Nas estações estudadas, as algas ocorreram em diferentes tipos de substratos (Tab. 2). O maior número de rodofíceas ocorreu como epilíticas, epífitas e epizóicas, totalizando 20 registros. Destes, as epífitas ocorreram em maior número, com 15 espécies. Neste substrato, a ocroficea Dictyota menstrualis (Hoyt) Schnetter, Hörnig, \& Weber-Peukert foi a hospedeira que apresentou o maior número de rodofíceas epífitas, seguida pelas rodofíceas Gelidiopsis variabilis (Greville ex J. Agardh) F. Schmitz e Nitophyllum wilkinsoniae Collins \& Herv. No substrato artificial ocorreram nove representantes, com oito registros em corda. O substrato rochoso, apesar de ser considerado o mais favorável para a fixação e desenvolvimento das algas bentônicas (Pereira 2000), no arquipélago encontrou-se recoberto, em sua maioria, pelas clorofíceas Caulerpa racemosa var. peltata (J. V. Lamour.), Bryopsis plumosa (Huds.) C. Agardh e B. pennata J. V. Lamour., mantendo a fisionomia observada por Edward \& Lubbock (1983).

As espécies de rodofíceas ocorreram em maior número na Estação 01 (Enseada), com 16 espécies, seguida pelas Estações 03 (Paredão da Ilha Challenger), com sete espécies, e a Estação 04 (Paredão da Ilha Cabral), com cinco espécies (Tab. 2). Nas Estações 02, 05 e 06 não ocorreram rodofíceas. Apesar da maior diversidade ter sido registrada na Estação 01, não há uma grande diversidade de macro-espécies neste local assim como em todo o Arquipélago. A área é coberta por tufos de algas filamentosas pertencentes aos gêneros $\mathrm{Ce}$ - 
Tabela 1. Sinopse das espécies de rodofíceas ocorrentes no Arquipélago de São Pedro e São Paulo, Brasil (Hemsley 1885; Edwards \& Lubbock 1983; Pereira et al. 2005 e no presente trabalho).

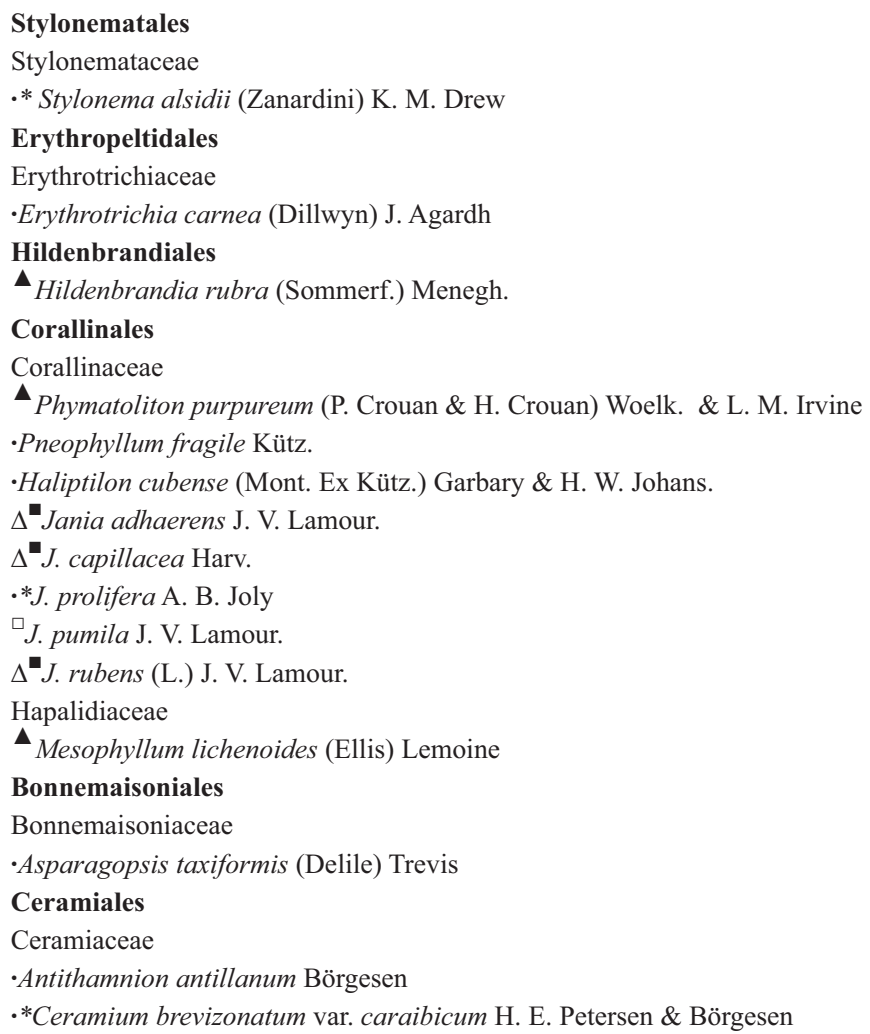

-Ceramium dawsonii A. B. Joly

-Ceramium flaccidum (Kütz.) Ardiss

- Ceramium luetzelburgii O.C. Schmidt

* Ceramium tenerrimum (G. Martens) Okamura

Delesseriaceae

$\Delta$ Hypoglossum hypoglossoides (Stackh.) Collins \& Herv.

$\Delta$ Nitophyllum wilkinsoniae Collins \& Herv.

Rhodomelaceae

${ }^{-}$Laurencia caraibica P. C. Silva

$\Delta$ L. filiformis (C. Agardh) Mont.

${ }^{\square}$ L. microcladia Kütz.

$\Delta$ Neosiphonia ferulacea (Suhr exs J. Agardh) S. M Guim. \& M.

I. Fujii

-Neosiphonia tepida (Hollenb.) S. M. Guim. \& M I. Fujii

$\triangle$ Polysiphonia subtilissima Mont.

\section{Gelidiales}

Gelidiaceae

-Gelidium pusillum (Stackh.) Le Jolis var. pusillum

Rhodymeniales

Champiaceae

$\Delta$ Champia parvula (C. Agardh) Harv.

Lomentariaceae

${ }^{\square}$ Gelidiopsis planicaulis (W.R. Taylor) W.R. Taylor

•G. variabilis (Grev. ex J. Agardh) F. Schmitz

* Nova adição para as ilhas oceânicas brasileiras.

- Nova adição ao Arquipélago de São Pedro e São Paulo.

\ Hemsley 1885

$\square$ Edwards \& Lubbock 1983

- Pereira et al. 2005

$\Delta$ Presente trabalho

ramium Roth, Polysiphonia Grev., Neosiphonia M. S. Kim \& I. K. Lee e calcárias geniculadas como Jania J. V Lamour. Em função do posicionamento geográfico, estrategicamente entre os dois hemisférios e ainda entre os continentes sulamericano e africano, o ASPSP é considerado um oásis no meio do oceano, exercendo uma forte influência no ciclo de vida de várias espécies migratórias, tais como peixes, crustáceos e aves que utilizam a região do arquipélago e seu entorno como zona de alimentação, reprodução e abrigo (Campos et al. 2005). Sendo assim a pressão exercida pela predação pode ser um dos fatores responsáveis pela mudança da comunidade, uma vez que foi observada a ocorrência de herbivoria, por peixes, o que poderia ter modificado a paisagem original. Segundo Cheroske et al. (2000) e Steneck \& Dethier (1994) o aumento na intensidade de herbivoria muda a comunidade de algas, sendo substituídas de macroalgas frondosas por tufos de filamentos de rápido crescimento, seguido de um desenvolvimento sucessional por outras formas de algas, como calcárias e foliáceas.
Com relação à ausência de rodofíceas nas Estações 02, 05 e 06, observou-se na Estação 02 uma zona de forte arrebentação e tal fato pode ter influenciado negativamente, na fixação das espécies. Nesta estação, ocorreram tapetes da feofícea Asteronema breviarticulatum Delépine \& Asensi. Segundo Széchy \& Cordeiro-Marino (1991), esta espécie é comumente encontrada em ambiente batido. A Estação 05 é, aparentemente, um local com alta herbivoria tendo sido observado apenas a ocorrência da clorofícea Caulerpa racemosa var. peltata (J. V. Lamour.) Eubank. A ocorrência alta de herbívoros é um conhecido fator negativo para a fixação das algas, pois a predação é uma fonte de distúrbio (Hay et al. 1983; Hixon 1991; McClanahan 1997; Morrison 1988). A herbivoria possui influência direta ou indireta na abundância de espécie das algas e grande parte de sua produção é consumida por herbívoros locais (Carpenter 1986; Hay et al. 1983; Morrison 1988). Peixes ou ouriços sozinhos são capazes de remover quase $100 \%$ da produção diária (Carpenter 1986; Hay 1991; 1996), e esta forte relação entre produção e 


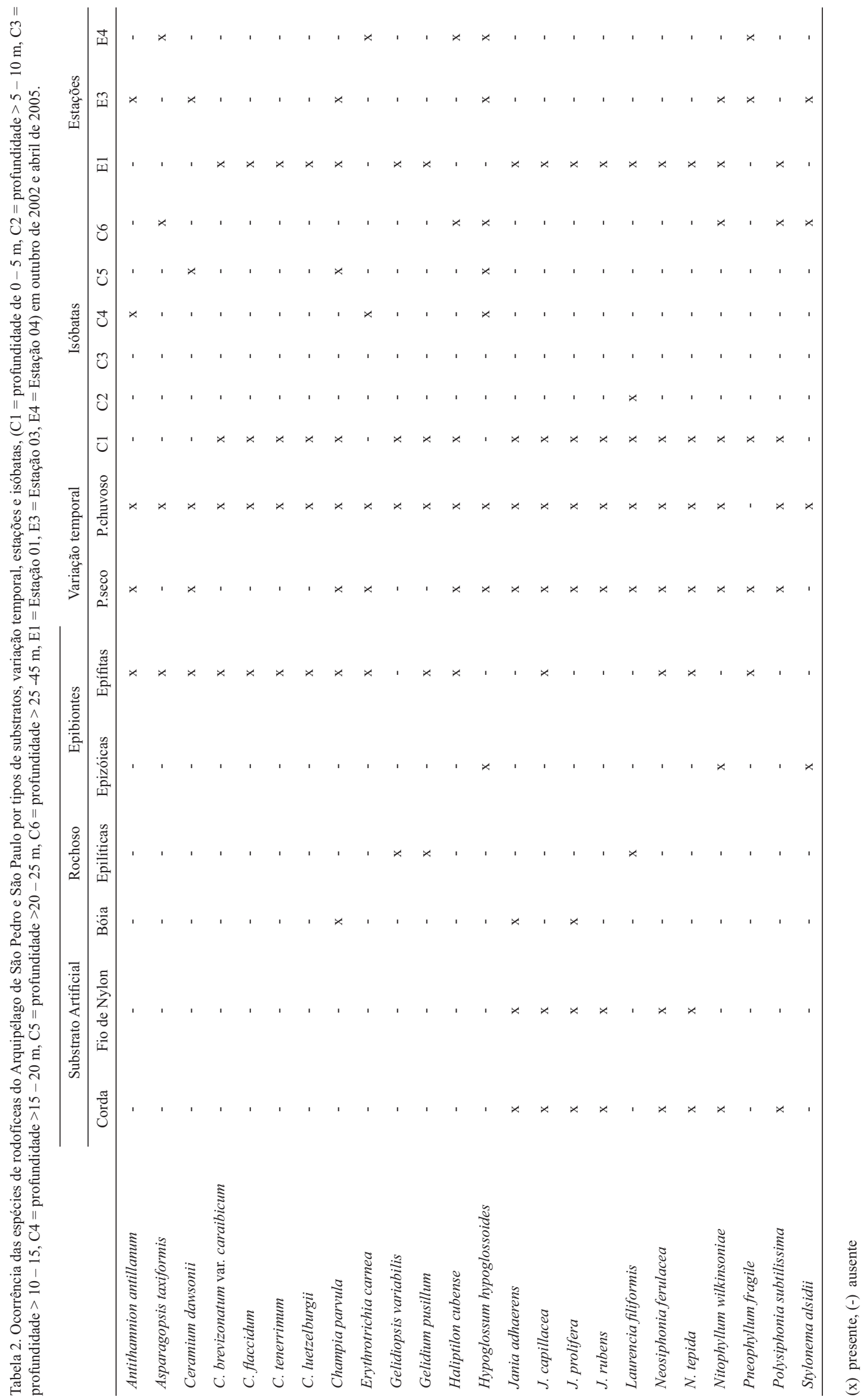


consumo, nos vários compartimentos recifais, faz com que a interação planta-herbívoro seja um dos mais importantes fatores, podendo modificar inteiramente a estrutura da comunidade (Boaventura et al. 2002). Finalmente na Estação 06, como foi referido anteriormente, notou-se o predomínio de Palythoa caribeorum Duchassaing \& Michelotti ocorrendo, apenas, a forma juvenil da clorofícea Caulerpa racemosa var. peltata. Este zoantídeo é um agressivo competidor por espaço, com taxa de crescimento entre $2.5-4.0 \mathrm{~mm} / \mathrm{dia}$, representando uma das mais elevadas taxas de crescimento conhecida para um anthozoa. Esta característica o coloca no alto de uma hierarquia competidora para ecossistemas recifais, tornando-o responsável pelo não desenvolvimento das macroalgas uma vez que ele recobre todo o substrato impedindo a fixação das macroalgas e de outros organismos (Suchanek \& Green 1981).

As rodofíceas não apresentaram um padrão de distribuição nos ambientes estudados. Das seis estações plotadas apenas três registraram a ocorrência desses representantes, mesmo assim nenhuma espécie foi comum entre estas estações (Tab. 2). Algumas algas foram exclusivas entre as estações. Na Estação 01 ocorreram como exclusivas Ceramium brevizonatum var. caraibicum, C. flaccitum, $C$. luetzelburgii, C. tenerrimum, Gelidiopsis variabilis, Gelidium pusillum, Jania adhaerens, J. capilacea, J. prolifera, J. rubens, Laurencia filiformis, Neosiphonia ferulacea, $N$. tepida e Polysiphonia subtilissima. Na Estação 03 Antithamnion antillanum, Ceramium dawsonii e Stylonema alsidii e na Estação 04 Asparagopsis taxiformis, Erythrotrichia carnea e Haliptilon cubense. As espécies Champia parvula e Nitophyllum wilkinsoniae foram comuns as Estações 01 e 03, enquanto que Hypoglossum hypoglossoides e Pneophyllum fragile foram comuns nas Estações 03 e 04.

Foi observada uma variação batimétrica na ocorrência das rodofíceas. Nas Estações 03 e 04 (Ilhas Challenger e Cabral) esses representantes ficaram restritos a Zona Subcaulerpa, numa faixa de profundidade entre $20-40 \mathrm{~m}$. As rodofíceas não ocorreram em menores profundidades devido à marcada presença de Caulerpa racemosa var. peltata nos paredões das respectivas ilhas, aparentemente limitando o espaço, por competição. Na Estação 01 (Enseada), as rodofíceas ocorreram sobre algas nas isóbatas de 5-6 m, não alcançando maiores profundidades, devido a zonação do ambiente, onde até os $33 \mathrm{~m}$ ocorre Caulerpa racemosa var. peltata, como observado anteriormente neste local por Edwards \& Lubbock (1983). Estes autores verificaram a presença do zoantídeo Palythoa caribeorum na isóbatas de 1-3 m. A ocorrência das rodofíceas na profundidade de $1 \mathrm{~m}$ foi verificada apenas em substrato artificial, corda (Tab. 2).

Com relação a variação temporal, observou-se que em relação ao de número de espécies não houve diferença significativa $\left(\chi^{2}=0,53 ; p=0,06\right)$ entre os períodos seco $(16$ espécies) e chuvoso (23 espécies) (Tab.2), mas a composição florística variou com sete novas espécies no período chuvoso. A ausência de informações sobre as variações sazonais da flora de ilhas oceânicas impossibilita a discussão se esta tendência é uma característica comum em ilhas ocêanicas, em resposta a variações climáticas.

A similaridade florística entre as profundidades das estações de coleta variou de 0 a $0,66 \%$ pelo índice de S申rensen (Tab.3). Os maiores percentuais de similaridades podem ser explicados, principalmente, pela baixa riqueza de espécie que ocorreu entre as profundidades, variando de 0 a 3 por batimetria em cada estação, exceção feita na estação E1C1 que apresentou 16 espécies.

A estação E1C1, como já comentado, representou um sítio especial para ocorrência das algas no Arquipélago, por reunir um número mais diversificado de microhabitats, ser mais protegida da forte arrebentação e da possível herbivoria por peixes, o que corrobora com a hipótese, sobre o fato da variedade de nichos favorecer o aumento da diversidade (Crawley 1989). No caso deste estudo verificou-se um aumento local, tendo em vista que a ilha como um todo apresentou baixa riqueza de táxons, enquadrando-se na teoria da biogeografia de ilhas ao admitir que ilhas pequenas e distantes de áreas contaminantes exibem baixa riqueza de espécies (Mac Arthur \& Wilson 1967). Como este trabalho é um dos poucos a utilizar o mergulho autônomo para estudar exclusivamente a ficoflora da região de infralitoral de ilhas oceânicas no Brasil, sugere-se que novas pesquisas sejam realizadas, visando confirmar a tendência quanto à diversidade de espécies nestes habitats, possibilitando melhor discussão sobre a teoria que melhor explica diversidade de espécies em área insular.

Tabela 3. Semelhança florística entre as estações e profundidades no conjunto dos períodos seco (outubro/2002) e chuvoso (abril/2005) $(E 1=$ Estação 01, E3 = Estação 03, E4 = Estação $04, \mathrm{C} 1=$ profundidade de $0-5 \mathrm{~m}, \mathrm{C} 4=$ profundidade $>15-20 \mathrm{~m}, \mathrm{C} 5=$ profundidade $>20-25 \mathrm{~m}, \mathrm{C} 6=$ profundidade $>25-45 \mathrm{~m})$.

\begin{tabular}{|c|c|c|c|c|c|c|c|}
\hline & E1C1 & E3C5 & $\mathrm{E} 4 \mathrm{C} 4$ & E4C6 & E3C4 & E4C5 & E3C6 \\
\hline \multicolumn{8}{|l|}{ E1C1 } \\
\hline E3C5 & 0,10 & & & & & & \\
\hline $\mathrm{E} 4 \mathrm{C} 4$ & 0 & 0,50 & & & & & \\
\hline E4C6 & 0 & 0,33 & 0,40 & & & & \\
\hline $\mathrm{E} 3 \mathrm{C} 4$ & 0 & 0,40 & 0,50 & 0,40 & & & \\
\hline $\mathrm{E} 4 \mathrm{C} 5$ & 0 & 0,50 & 0,66 & 0,50 & 0,66 & & \\
\hline E3C6 & 0,10 & 0,33 & 0,40 & 0,33 & 0,40 & 0,33 & \\
\hline
\end{tabular}


Comparando-se a flora de rodofíceas do ASPSP com as de outras ilhas oceânicas brasileiras, observa-se que o referido arquipélago apresentou menor número de táxons (Fig. 4), quando comparado ao registrado nos arquipélagos de Fernando Noronha, Trindade e Martin Vaz e no Atol das Rocas (Villaça et al. 2006). Não foi possível analisar, separadamente, a diversidade específica das algas dos arquipélagos Trindade e Martins Vaz porque Yoneshigue-Valentin et al. (2005) ao realizarem o levantamento florístico, consideraram os referidos arquipélagos como um único complexo insular.

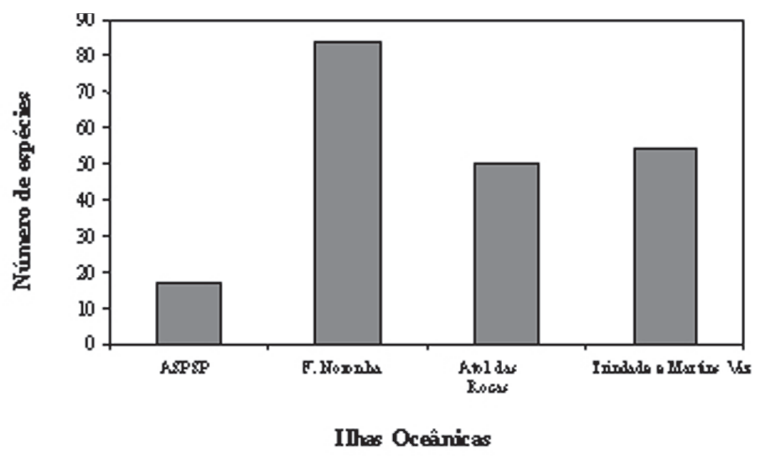

Figura 4. Número de espécies de rodofíceas das ilhas oceânicas brasileiras.

A análise de agrupamento permitiu identificar a existência de apenas um grupo florístico consistente, formado pelo litoral de Pernambuco, Arquipélagos de Fernando Noronha e de Trindade (Fig. 5), indicando a existência de possíveis correlações entre a flora de rodofíceas destes locais. No entanto fica inviabilizada uma análise mais consistente desse aspecto, baseando-se, apenas, na ocorrência de espécies em comum destas ilhas. Seria necessário o estudo do sistema de correntes e outros fatores biológicos, como dispersão e resistência de esporos e zigotos, para se estabelecerem e definir o grau de conectividade (Cowen et al. 2000, 2007). A ligação florística entre as demais localidades não foi significativa pelo teste de permutação Monte Carlo $(p=0,05)$, não permitindo uma melhor abordagem.

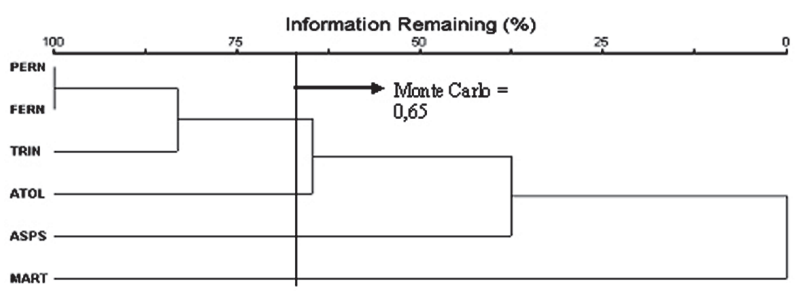

Figura 5. Similaridade florística das rodofíceas entre as ilhas oceânicas brasileiras e o litoral do Estado de Pernambuco $($ PERN = Pernambuco; FERN = Arquipélago de Fernando de Noronha; TRINT $=$ Arquipélago de Trindade; $\mathrm{ATOL}=$ Atol das Rocas; ASPSP = Arquipélago de São Pedro e São Paulo e MART = Arquipélago de Martin Vaz).

\section{Agradecimentos}

Os autores agradecem à Secretaria da Comissão Interministerial para Recursos do Mar (SECIRM) e Marinha do Brasil pelo apoio financeiro e logístico. Ao Conselho Nacional de Desenvolvimento Científico e Tecnológico (CNPq), pelo auxílio financeiro ao projeto e pelas concessões das Bolsas de Mestrado ao primeiro autor e de Produtividade de Pesquisa ao segundo autor.

\section{Referências bibliográficas}

Bandeira-Pedrosa, M.E., Bouzon, Z.L., Pereira, S.M.B. \& Oliveira, E. C. 2003. Ultrastructure of some species of Halimeda (Bryopsidales, Chlorophyta) from Brazil. Cryptogamie Algologie 24(3): 219-231.

Bandeira-Pedrosa, M.E.; Pereira, S.M.B. \& Oliveira, E.C. 2004. Taxonomy and distribution of the green algal genus Halimeda (Bryopsidales, Chlorophyta) in Brazil. Revista de Brasileira Botânica 27(2): 363-367.

Barbosa, J.P.; Texeira, V.L.; Villaça, R.; Pereira, R.C.; Abrantes, J.L. \& Frugulhetti, I.C.P.P. 2003. A dolabellane diterpene from the brazilian brown alga Dictyota pfaffii. Biochemical Systematics and Ecology 31: 1451-1453.

Boaventura, D.; Alexander, M.; Della Santina, P.; Smith, N.D.; Ré, P.; da Fonseca, L.C. \& Hawkins, S.J. 2002. The effects of grazing on the distribuiçào and composition of low-shore algal communities on the central coast of Portugal and on the southern coast of Britains. Journal of Experimental Marine Biology and Ecology 267(2): 185-206.

Campos, T.F.D.; Virgens-Neto, J.; Srivastava, N.K.; Petta, R.A.; Hartmann, L.A.; Moraes, J.F.S.; Mendes, L. \& Silveira, S.R.M. 2005. Arquipélago de São Pedro e São Paulo - Soergimento tectônico de rochas ingracrustais no Oceano Atlântico. In M. Winge; C. Schobbenhaus; M. Berbert-Born; E.T. Queiroz; D. A. Campos; C.R.G. Souza \& A.C.S. Fernandes (org.) Sítios Geológicos e Paleontológicos do Brasil. SIGEP 002, 12p http://www.unb.br/ig/sigep/sitio002/sitio002.pdf. acessado $12 / 11 / 2008$.

Carpenter, R.C. 1986. Partitioning herbivory and its effects on coral reef algal communities. Ecological Monographs 56(4): 345-363.

Cheroske, A.G.; Williams, S.L. \& Carpenter, R.C. 2000. Effects of physical and biological distubances on algal turfs in Kaneohe Bay, Hawaii. Journal of Experimental Marine Biology and Ecology 248 (1): 1-34.

Cowen, R.K.; Lwiza, K.M.M.; Sponaugle, S.; Paris, C. B. \& Olson, D. B. 2000. Connectivity of Marine Populations: Open or Closed?. Science 287: 857-859.

Cowen, R.K.; Gawarkiewich, G.; Pineda, J.; Thrrold, S.R. \& Werner, F.E. 2007. Population Connectivity in Marine Systems An Overview. Oceonography 20(3): 14-21.

Crawley, M. 1989. The successes and failures of weed biocontrol using insects. Biocontrol News and formation 10: 213-223.

Dickie, G. 1875. Enumeration of algae from Fernando de Noronha collected by H. N. Moseley, M. A., naturalist to H. M. S. Challenger. Journal Linnean Society Botany 14(77): 355-359.

Edwards, A.J. 1984. The marine biology of Saint Paul's Rocks, Equatorial Atlantic Ocean. In: Occasional Papers in Biology 9: 594/ 75, Memorial University of Newfoundland.

Edwards, A. \& Lubbock, R. 1983. The ecology of Saint Paul's Rocks (Equatorial Atlantic). Journal of zoology 200: 51-69.

Feitosa, B.M.; Rocha L.A.; Luiz-Junior, O.J.; Floeter, S.R. \& Gasparini, J.L. 2003. Reef fishes of St. Paul's Rocks: new records and notes on biology and zoogeography. Journal of Ichthyology and Aquatic Biology 7(2): 61-82.

Fujii, M.T. \& Villaça, R. 2003. On the ocurrence of Laurencia caraibica (Ceramiales, Rhodophyta) in Atol das Rocas, Brazil. Hidrobiologica 13(1): 33-38.

Guimarães, S.M.P.B. 2006. A revised checklist of benthic marine Rhodophyta from the State of Espírito Santo, Brazil. Boletim do Instituto de Botânica 17: 143-194.

Guimarães, S.M.P.B.; Cordeiro-Marino, M \& Yamaguishi-Tomita, N. 1981. Deep water Phaeophyceae and their epiphytes from northeastern and southeastern Brazil. Revista Brasileira de Botânica 4: 95-131.

Hay, M.E. 1991. Fish-seaweed interactions on coral reefs: Effects of herbivorous fishes and adaptations of their prey. Pp. 96-119. In: P.F. Sale (ed.). The Ecology of Fishes on Coral Reefs. San Diego, Academic Press, Inc.

Hay, M.E. 1996. Marine chemical ecology: What's know and what's next? Journal of Experimental Marine Biology and Ecology 200(1-2): 103-134.

Hay, M.E.; Colburn, T \& Downing, D. 1983. Spatial and temporal patterns in herbivory on a Caribbean fringing reef: the effects on plant distribuition. Oecologia 58(3): 299-308.

Hemsley, W.B. 1885. Report on the botany of the Bermudas and various other 
islands of the Atlantic and southern oceans. Report on the Scientific Results of the Voyage of H.M.S. Challenger. Botany 1(2): 1-135, pls 1-13.

Hixon, M.A. 1991. Predation as a process struturing coral reef fish communities. Pp. 475-508. In: P.F. Sale (ed.). The Ecology of Fishes on Coral Reefs. San Diego, Academic Press, Inc.

Joly, A.B. 1950. Resultados científicos do cruzeiro do "Baependi" e do "Veja á Ilha da trindade - Nota Preliminar sobre algumas algas. Boletim do Instituto Oceanográfico da Universidade de São Paulo 1(2): 73-75.

Joly, A.B. 1953. Scientific results of the "Baependi" and "Veja" Cruise to the Trindade Island. Boletim do Instituto Oceanográfico da Universidade de São Paulo 4: 147-156.

Joly, A.B. \& Oliveira Filho, E. C. 1968. Notes on Brazillian algae II. A new Anadyomene of the deep water flora. Phycus 7(1): 27-31.

Joly, A.B. \& Sazima, M. 1971. Brazillian deep water marine algae. Additions to the Brazillian Flora. III. Ciência e Cultura 23(3): 333-336.

Joly, A.B.; Sazima, M. \& Semir, J. 1976. Brazillian deep water marine algae. Additions to the Brazillian Flora. IV. Boletim de Botânica da Universidade de São Paulo 4: 71-91.

Joly, A.B. \& Yoneshigue-Braga, Y. 1966. Primeira nota sobre algas marinhas durante as viagens do navio oceanográfico Almirante Saldanha. Instituto de Pesquisas Marinhas 34: 103-108.

Lubbock, H. R. \& Edwards, A. J. 1981. The fishes of St. Paul's Rocks. Journal of Fish Biology 18: 135-137.

MacArthur, R.H. \& Wilson, E.O. 1967. The theory of island biogeography. Princenton University Press.

Manly, B.F.I. 1997. Randomization bootstrap and Monte Carlo methods in biology. New Zealand, Chapman \& Hall.

Mansilla, A.O.M. \& Pereira, S.M.B. 1998. Variação temporal da abundância e composição específica da macroflora associada a uma população de Sargassum (Fucophyceae do litoral de Pernambuco, Brasil). Boletim de Botânica da Universidade de São Paulo 17: 271-276.

McClanahan, T.R. 1997. Primary sucession of coral reef algae: Differing patterns on fished versus unfished reefs. Journal of Experimental Marine Biology and Ecology 218(1): 77-102.

McCune, B. \& Mefford, M.J. 1999. PC-ORD version 4.0, multivariate analysis of ecological data, Users guide. Glaneden Beach. MjM Software Design,

Morrison, D. 1988. Comparing fish and urchin grazing in shallow and deeper coral reef algal communities. Ecology 69(5): 1367-1382.

Muñoz, A.O.M. \& Pereira, S.M.B. 1997. Caracterização quali-quantitativa das comunidades de macroalgas nas formações recifais da Praia do Cupe -Pernambuco (Brasil). Trabalhos Oceanográficos da Universidade Federal de Pernambuco 25: 3-109.

Murray, G. 1891. Algae. In H. N. Riddley: Notes on the botany of Fernando de Noronha. Journal Linnaeus Society (Botany) 27: 75-80.

Oliveira-Carvalho, M.F., Pereira, S.M.B. \& Zickel, C.S. 2003. Florística e Distribuição espaço-temporal das Clorofíceas bentônicas em trechos recifais do litoral Norte do Estado de Pernambuco - Brasil. Hoehnea 30(3): 201-212.

Oliveira Filho, E.C. 1969. Algas marinhas do sul do Estado de Espírito Santo (Brasil). I - Ceramiales. Boletim da Faculdade de Filosofia e. Ciências da Universidade de São Paulo (ser. Bot.) 26: 1- 277.

Oliveira Filho, E.C. 1976. Deep water marine algae from Espírito Santo State (Brazil). Boletim de Botânica da Universidade de São Paulo 4: 73-80.

Oliveira Filho, E.C. \& Ugadim, Y. 1974. New References of Benthic Marine Algae to Brazilian Flora. Boletim de Botânica da Universidade de São Paulo 2: 71-91

Oliveira Filho, E. C. \& Ugadim, Y. 1976. A survey of the marine algae of Atol das Rocas (Brazil). Phycologia 15(1): 41-44.

Pedrini, A.G.; Gonçalves, E.A.; Fonseca, M.C.S.; Zaú, A.S. \& Lacorte, C.C. 1989. A survey of the marine algae of Trindade Island, Brazil. Botanica Marina 32: 97-99.

Pedrini, A.G.; Ugadim, Y.; Braga, M.R. \& Pereira, S.M.B. 1992. Algas marinhas bentônicas do Arquipélago de Fernando de Noronha, Brasil. Boletim de Botânica da Universidade de São Paulo 13: 93-101.

Pereira, S.M.B. 2000. As algas bentônicas. Pp. 49-65. In: H.M. Barros; E. Eskinazi-Leça; S. J. Macedo; T. Lima. (org.). Gerenciamento participativo de estuários e manguezais 1. Recife, Editora Universitária da Universidade Federal de Pernambuco.
Pereira, S.M.B. 2006. Algas marinhas bentônicas do Arquipélago de Fernando de Noronha (PE) e Arquipélago de São Pedro e São Pulo. Pp. 113-126. In: R.J.V. Alves \& J.W.A. Castro (eds). Ilhas Oceânicas Brasileiras da Pesquisa ao Manejo. Cap. 5, $2^{\text {a }}$ parte. Brasília, Ministério do Meio Ambiente.

Pereira, S.M.B \& Accioly, M.C. 1998. Clorofíceas marinhas bentônicas da Praia de Serrambi - Pernambuco. Acta Botânica Brasílica 12: 29-56.

Pereira, S.M.B.; Bandeira-Pedrosa, M.E. \& Burgos, D.C. 2005. Macroalgae from the Saint Peter's and Saint Paul's Archipelago (Brazil). Phycologia 36(4): Supplement. p. 80.

Pereira, S.M.B.; Burgos, D.C. \& Bandeira-Pedrosa, M.E. 2006. Representantes da Flora. Pp.36-41. In: Vaske-Jr.; R.P. Lessa; M.F. Nóbrega; F.M.D. Amaral \& S. R. M. Silveira. (eds.). Arquipélago de São Pedro e São Paulo: Histórico e Recursos Naturais 1. Olinda, Livro Rápido - Ed. Elógica

Pereira, S.M.B.; Mansilla, A.O.M. \& Cocentino, A.L.M. 1996. Ecological aspects of a benthic marine algal community in southeast bay, Archipelago of Fernando de Noronha, Brazil. Trabalhos Oceanográficos da Universidade Federal de Pernambuco 24: 157-163.

Pereira, S.M.B.; Oliveira-Carvalho, M.F.; Angeiras, J.A.P; BandeiraPedrosa, M.E.; Oliveira, N.M.B.; Torres, J.; Gestinari, L.M.S.; Cocentino, A.L.M.; Santos, M.D.; Nascimento, P.R.F. \& Cavalcanti, D.R. 2002. Algas marinhas bentônicas do estado de Pernambuco. Pp. 97-124. In: M.Tabarelli, J.M.C. Silva (org.). Diagnóstico da Biodiversidade de Pernambuco 1. Ed. Massangana, Recife.

Pereira, S.M.B.; Oliveira-Carvalho, M.F.; Burgos, D.C. \& Araújo, E. de L. 2008. Caracterização estrutural das macroalgas de ambiente recifal da praia de enseada dos corais - PE - Brasil. In: CONGRESSO BRASILEIRO DE FICOLOGIA, 11.; SIMPÓSIO LATINOAMERICANO SOBRE ALGAS NOCIVAS, 2006, Itajaí, SC. Aplicações da Ficologia: anais... Rio de Janeiro: Museu Nacional. p.231-242. Organização da Sociedade Brasileira de Ficologia. (Série Livros, 30).

Pereira, S.M.B.; Oliveira Filho, E. C.; Araújo, M.S.B de; Paes e Mello, L.B.; Carvalho, F.A.F. de \& Câmara Neto, C. 1981. Prospecção dos bancos de algas marinhas do Estado do Rio Grande do Norte. $2^{\mathrm{a}}$. Parte: Profundidade de 10 a 45 metros. Série: Brasil. SUDENE. Estudos de Pesca 9: 27-81.

Richardson, W.D. 1975. The marine algae of Trinidad, West Indies. Bulletin of the British Museum (Natural History) Botany 5(3): 96-107.

Steneck, R.S \& Dethier, M.N. 1994. A functional group approach to the structure of alga-dominated communities. Oikos 69(3): 476-498.

Suchanek, T.H. \& Green, D.J. 1981. Interspecific competition between Palythoa caribaeorum and other sessile invertebrates on St. Croix Reefs, U. S. Virgin Islands. Proceedings of the Fourth International Coral Reef Symposium 2: 679-684.

Széchy, M.T.M. \& Cordeiro-Marino, M. 1991. Feofíceas do litoral norte do Estado do Rio de Janeiro, Brasil. Hoehnea 18: 205-241.

Széchy, M.T.M.; Maurat, M.C.S; Nassar, C.A.G. \& Falcão, C. 1987. Adições à flora marinha bentônica do Arquipélago de Fernando de Noronha. Nerítica 2: 135-146.

Széchy, M.T.M.; Nassar, C.A.G.; Falcão, C. \& Maurat, M.C.S. 1989. Contribuição ao inventário das algas marinha bentônicas de Fernando de Noronha. Rodriguesia 67(41): 53-61.

Torres, T.; Pereira, S.M.B. \& Yoneshigue-Valentin,Y. 2004. Ceramiaceae (Rhodophyta) de áreas recifais do estado de Pernambuco. Hoehnea 31(3): 201-212.

Travassos, P.; Hazin, F.H. \& Zagaglia, J.R. 1999. Thermohaline structure around seamounts and islands off north-eastern Brazil. Archive of Fishery Marine Resarch 47(2/3): 211-222.

Ugadim, Y \& Pereira, S.M.B. 1978. Deep-water marine algae from Brazil collected by the Recife Comission. I - Clorophyta. Ciência e Cultura 30(7): 838-842.

Vaske-Jr. T; Lessa, R.P.; Nóbrega, M.F; Montealegre-Quijano, S.; Marcante Santana, F. \& Bezerra Jr., J.L. 2005. A checklist of fishes from Saint Peter and Saint Paul Archipelago, Brazil. Journal Applied Ichthyology 21: $75-79$

Vaske-Jr. T.; Nóbrega, M.F.; Santana, F.M.; Silveira, S.R.M. \& Amaral, F.M.D. 2006. Características locais. Pp. 14-23. In: T. Vaske-Jr.; R.P. Lessa; M.F. Nóbrega; F.M.D. Amaral \& Silveira, S.R.M. (eds.) 
Arquipélago de São Pedro e São Paulo: Histórico e Recursos Naturais 1. Olinda, Livro Rápido - Ed. Elógica.

Villaça, R.; Pedrini, A.G.; Pereira, S.M.B. \& Figueiredo, M.A.O. 2006. Flora marinha bentônica das ilhas oceânicas brasileiras. Pp. 106-146. In: R.J.V. Alves \& J. W. de A. Castro (org.) Ilhas Oceânicas Brasileiras da Pesquisa ao Manejo. Brasília. Ministério do Meio Ambiente (MMA).

Yoneshigue-Braga, Y.; Boudouresque, C.F. \& Figueiredo, M.A.O. 1986. Flore algale marina de la region de Cabo Frio, état de Rio de Janeiro (Brésil). 5. - Sur Boodlea compósita (Boodleaceae - chlorophyta), Dictyota pardalis (Dictyota - Phaeophyta) et Lophosiphonia cristata (Rhodomelaceae - Rhodophyta), espéces nouvelles pour la cote brésilenne. Rickia, São Paulo, p. 17-27.

Yoneshigue-Valentin, Y; Fernandes, D.R.P.; Pereira, C.B. \& Ribeiro,
S.M. 2005. Macroalgas da Plataforma Continental da Ilha da Trindade e do Arquipélago de Martin Vaz (Espírito Santo Brasil) Pp. 361-372. In: Anais da X Reunião Brasileira de Ficologia. Formação de Ficólogos: um compromisso com a sustentabilidade dos recursos aquáticos. Salvador 2004. Rio de Janeiro, Museu Nacional. P. Org. Sociedade Brasileira de Ficologia (série livros; 10).

Williams, L.G. \& Blomquist, H.L. 1947. A collection of marine algae from Brazil. Bulletin of the Torrey Botany Club 5: 383-397.

Wynne, M.J. 2005. A checklist of benthic marine algae of the tropical and subtropical western atlantic: second revision. Nova Hedwigia 129: 1-152.

Zar, J. 1996. Biostatistical analysis. (2 $2^{\text {nd }}$ edition). New Jersey, Prentice Hall. 